\title{
Review of Pure Endoscopic Full-Thickness Resection of the Upper Gastrointestinal Tract
}

\author{
Hirohito Mori, Hideki Kobara, Noriko Nishiyama, Shintaro Fujihara, and Tsutomu Masaki \\ Department of Gastroenterology and Neurology, Kagawa University, Kita, Japan
}

Natural-orifice transluminal endoscopic surgery (NOTES) using flexible endoscopy has attracted attention as a minimally invasive surgical method that does not cause an operative wound on the body surface. However, minimizing the number of devices involved in endoscopic, compared to laparoscopic, surgeries has remained a challenge, causing endoscopic surgeries to gradually be phased out of use. If a flexible endoscopic full-thickness suturing device and a counter-traction device were developed to expand the surgical field for gastrointestinal-tract collapse, then endoscopic full-thickness resection using NOTES, which is seen as an extension of endoscopic submucosal dissection for full-thickness excision of tumors involving the gastrointestinal-tract wall, might become an extremely minimally invasive surgical method that could be used to resect only full-thickness lesions approached by the shortest distance via the mouth. It is expected that gastroenterological endoscopists will use this surgery if device development is advanced. This extremely minimally invasive surgery would have an immeasurable impact with regard to mitigating the burden on patients and reducing healthcare costs. Development of a new surgical method using a multipurpose flexible endoscope is therefore considered a socially urgent issue. (Gut Liver 2015;9:590-600)

Key Words: Minimally invasive surgical procedures; Suturing; Traction; Micro-Electrical-Mechanical Systems

\section{INTRODUCTION}

The history of treatment employing flexible endoscopes started with the advent of polypectomy as a procedure for removing small polyps. Subsequently, flexible endoscopy evolved to endoscopic mucosal resection with local injection. The procedure further evolved into an extremely minimally invasive treatment for gastrointestinal malignant tumors. This treatment is called endoscopic submucosal dissection (ESD) with electrocautery and is used exclusively with flexible endoscopes. The lesions that can be treated with ESD have been extended from the mucosa to the subserosa, while various ESD-related derivational procedures and inventions have been reported. ${ }^{1-3}$

Since Kalloo et al., ${ }^{4}$ at Johns Hopkins University, published a study involving observations of the abdominal cavity obtained through the stomach using oral flexible endoscopy following incision of the stomach wall in 2004, natural-orifice transluminal endoscopic surgery (NOTES) using flexible endoscopy has attracted attention as an extremely minimally invasive surgical method that results in no operative wounds on the body surface. The scope of treatment was immediately extended to diseases treated with laparoscopic surgery, resulting in various comparisons, including levels of invasion and safety concerns, between flexible endoscopic and laparoscopic surgical techniques. However, laparoscopy, which is well established, involves many devices. On the contrary, flexible endoscopy only requires incision and resection devices and several types of hemostats. There are many laparoscopic suturing devices, of which the autosuture laparoscopic stapler and laparoscopic hand-sewn method have been of particular interest. Many types of linear staplers and circular staplers are on the market, and a number of laparoscopic operative procedures have been reported..$^{5-11}$

Therefore, NOTES has particularities in its procedure that are unique to previous intraluminal therapies. Among these, the development of a full-thickness suturing device and the expansion of the surgical field should be considered most important. ${ }^{12-14}$ It is very difficult to rapidly succeed in developing a surgical method equivalent to laparoscopic surgery from the viewpoint of surgical safety and necessary devices. Thus, endoscopic fullthickness resection (EFTR), which is seen as an extension of ESD for full-thickness excision of tumors involving the gastrointes-

Correspondence to: Hirohito Mori

Department of Gastroenterology and Neurology, Kagawa University, 1750-1 Ikenobe, Miki, Kita 761-0793, Japan

Tel: +81-87-891-2156, Fax: +81-87-891-2158, E-mail: hiro4884@med.kagawa-u.ac.jp

Received on October 2, 2014. Accepted on May 7, 2015.

pISSN 1976-2283 eISSN 2005-1212 http://dx.doi.org/10.5009/gnl14380

(c) This is an Open Access article distributed under the terms of the Creative Commons Attribution Non-Commercial License (http://creativecommons.org/licenses/by-nc/4.0) which permits unrestricted non-commercial use, distribution, and reproduction in any medium, provided the original work is properly cited. 
tinal tract wall, has attracted attention recently, as evidenced by many published studies. ${ }^{15-18}$ EFTR is considered among the NOTES-related procedures, because the incision knife is required to reach outside of the stomach wall and into the abdominal cavity. If development of the device is advanced and operative procedures are established, gastroenterological endoscopists may find themselves performing EFTR surgeries in the future, and EFTR may become the extremely minimally invasive surgery of choice.

As experienced with NOTES, the development of systems that entirely change the endoscope itself, has been naturally rejected by endoscopists, resulting in the cessation of pursuing such developments. Based on this history and from the perspective of health economics, it is considered best that EFTR advancements employ existing devices for multipurpose flexible endoscopy as much as possible. As such, advancements have concentrated on reducing deficiency, and developing novel surgical procedures and surgical fields, rather than developing an entirely new EFTR procedure. Focusing on full-thickness excision of the gastrointestinal-tract wall and based on previous reports, the present study outlines the devices used for flexible endoscopy as well as the operative procedures and the latest devices employed in pure EFTR that exclusively utilizes flexible endoscopy using a prototype of the full-thickness suturing device under development. Future prospects are also discussed in this article.

\section{MATERIALS AND METHODS}

\section{Search strategy}

Relevant randomized studies were identified by searching the Cochrane Central Register of Controlled Trials, MEDLINE and EMBASE. Three authors (H.M., H.K., T.M.) independently inspected all identified references.

\section{Selection criteria for the study}

Studies were eligible if primary and secondary outcomes were clearly mentioned.

\section{Solutions sought for problems associated with pure EFTR}

Given the difficult problems reported in studies regarding NOTES or EFTR, it is essential to address the following considerations to make EFTR fit for safe and practical use: (1) target disease; (2) standardization of gastric lavage and disinfection for intra-abdominal infection caused by perforation of the stomach wall; (3) suturing of full-thickness wound after full-thickness excision (development of devices for flexible endoscopy and of full-thickness suturing device); (4) expansion of a surgical field for gastrointestinal-tract collapse caused by perforation (development of counter traction device); (5) monitoring of abdominal air pressure by insufflation (development of pressuremonitoring system); (6) training and future perspective of EFTR.

These considerations are outlined in more detail below.

\section{1) Target disease}

In previous reports, the most common indication for EFTR in humans was gastrointestinal stromal tumor (GIST). ${ }^{19-21}$ The reasons for this are as follows: the resection line could be identified from within the stomach; there was no risk of tumor dissemination; lymph node dissection was unnecessary; local excision could be performed relatively safely for the introduction of NOTES. For Pure EFTR, a GIST size of 3 to $4 \mathrm{~cm}$ was chosen so that lesions could be removed through the mouth. ${ }^{20}$

For local segmental resection of the stomach by laparoscopic surgery, a port, from which the full-thickness stomach wall would be resected, was placed on the abdominal wall, meaning that two sites (both abdominal and stomach walls) would be involved. One study report that EFTR is less invasive because only a full-thickness lesion is resected via approach from the mouth, the shortest distance, and because GIST is a good indication for EFTR ${ }^{17}$ Another study has reported that epithelial neoplasm, including gastric cancer, may become a future indication for EFTR if the problem regarding peritoneal metastasis of gastric cancer (sm massive: early gastric submucosal cancer defined as over $500 \mu \mathrm{m}$ deeper invasion from muscularis mucosa) is resolved and an operative procedure that does not expose a tumor within the abdominal cavity is established technically. ${ }^{16}$

\section{2) Standardization of gastric lavage and disinfection for intra-abdominal infection caused by perforation of the stomach wall}

Generally, hand washing before/during surgery is performed to reduce the normal level of bacteria, $10^{4-5}$ colony-forming units (CFU), to $10^{2-3} \mathrm{CFU}$, which is the level that can minimize contamination of the surgical field even if surgical gloves are damaged. In approaching a targeted organ via skin incision, such as surgery, pathogenic bacteria that can cause infection include resident flora, tubercle bacilli, filamentous fungus, spore forming bacteria, and viruses. The disinfection process employed for surgery is standard to control peritoneal infection. In other words, infection control as the minimum level is obtained by maintaining a clean area where the amount of viable fungi is not more than $10^{2-3} \mathrm{CFU}$.

In previous NOTES-related studies, Ryou et al. ${ }^{22}$ concluded the following from their experimental findings using swine: combination of both intravenous cefazolin and either chlorhexidine or povidone-iodine effectively reduced the bacterial load to the $10^{1} \mathrm{CFU} / \mathrm{mL}$ level in both gastric and colonic mucosa. However, there seem to be problems in their methodology. One hour after intravenous injection of cefazolin, the swine were sacrificed. The gastric mucosa and colonic mucosa were cut into $1.5-\mathrm{cm}$ segments, which were then irrigated with saline (the quantity of saline was not reported). Then, the mucosae were exposed to $4 \%$ chlorhexidine and 10\% povidone-iodine for 5 minutes to evaluate effects on the decrease in total bacterial count. Growth of bacteria began between the point in time when the swine 
were sacrificed and the point in time when the mucosae were cut into sections, making accurate bacterial cultures impossible. Unless in vivo animal experiments are performed, this study can be used only as a reference. Especially for the stomach, where gastric acid is present, the problem with routing through the stomach may be caused by resident flora in the oral cavity after the elimination of Helicobacter pylori. A large quantity of bacteria flows into the stomach when the mucosa goes through the mouth. Regarding the bacteria in the cut stomach in the aforementioned article, there is a large difference in the bacterial count and bacterial species obtained in an in vivo study compared to a study using the pathway via the mouth. Although the authors described that intravenous cefazolin and 4\% chlorhexidine or 10\% povidone-iodine would reduce the bacterial count to $10 \mathrm{CFU}$, intravenous cefazolin is commonly administered 30 minutes before normal surgery and inhibits postoperative infection. ${ }^{23,24}$ In the route via the stomach, it seems to be essential to administer cefazolin intravenously 30 minutes before surgery. The problem is that damage to human gastric mucosa by $4 \%$ chlorhexidine or $10 \%$ povidone-iodine was not taken into consideration. Although these agents sterilize bacteria, they also greatly damage the living body. Many studies reported that $10 \%$ povidone-iodine, in particular, causes severe skin disorders, such as burn on human skin. ${ }^{25-27}$ In addition, oral administration of $4 \%$ chlorhexidine and $4 \%$ chlorhexidine exposed to the stomach wall are contraindicated. Consequently, these authors' results were questionable with regard to whether their methods can be made safely available. It remained unclear whether the method was safe for the gastrointestinal tract, which is glandular epithelium rather than skin. Even if it can be used in animal experiments, their method is considered difficult to use in actual clinical settings. As much as 2 to $3 \mathrm{~L}$ of saline is used for irrigation during surgery, injury, and debridement, and saline's inhibitory effect on bacterial infection has been reported. There is also a report that irrigation using saline combined with antibiotics may provide protective efficacy in the treatment of peritonitis. ${ }^{28}$ Higher-pressure irrigation used with a flexible endoscope equipped with a water jet has also been reported to be effective. $^{29}$

Sodergren et al. ${ }^{30}$ went only so far as to conclude the following, based on the Oxford Centre for Evidence-Based Medicine guidelines: further human trials need to be conducted to corroborate the current evidence base for transgastric closure; it is important that future trials are conducted in a methodologically robust fashion, with emphasis on clinical outcomes. Data that are closest to that obtained in a clinical setting were shown in a study by Memark et al. ${ }^{31}$ involving transgastric endoscopic peritoneoscopy performed before 40 patients were scheduled for laparoscopic Roux-en-Y gastric bypass. According to this study, there was no significant difference between aspirate of saline within the stomach before surgery and cultivation of postoperative intra-abdominal saline aspiration; gastric aspi- rates comprised $980 \mathrm{CFU} / \mathrm{mL}(\mathrm{n}=40)$, while the median number of bacteria isolated from the peritoneal aspirates was $323 \mathrm{CFU} /$ $\mathrm{mL}$. In addition, according to their study, although the number of cultured bacteria increased in proton pump inhibitor (PPI) users, complications due to infection did not increase, because intravenous antibiotics were combined with PPI. ${ }^{31}$ Narula et al. ${ }^{32}$ reported a case of diagnostic laparoscopy for evaluation of presumed pancreatic cancer as follows: gastroscope aspirate was $132.1 \mathrm{CFU} / \mathrm{mL}$, peritoneal aspirates prior to creation of a gastrotomy showed $160.4 \mathrm{CFU} / \mathrm{mL}$, and peritoneal sampling after gastrotomy had an average of $642.1 \mathrm{CFU} / \mathrm{mL}$; there was no contamination of the peritoneal cavity with species. In the present study, we examined gastric lavage and disinfection for ESD which may be affected by the resident flora in the oral cavity.

There were ethical problems in the above-mentioned mucosa exposure of $4 \%$ chlorhexidine and $10 \%$ povidone-iodine because their safety in human gastric mucosa was not established, despite the fact that risk has been noted. The present authors performed a prospective study on the effect of gastric lavage with $2 \mathrm{~L}$ of saline in the lumen on the reduction of total bacterial count and then evaluated the effect in a semi-closed system. Because the endoscope is inserted in the stomach via the oral cavity, exposure to oral bacteria is inevitable. Fifty patients diagnosed with early gastric cancer were divided into the clean group (25 patients) and the regular group (25 patients). The day before surgery, $30 \mathrm{mg}$ of lansoprazole, a PPI, was administered once a day to both groups (all bacteria were cultured at $37^{\circ} \mathrm{C}$ for 48 hours). For bacterial culture in the stomach before gastric lavage, $20 \mathrm{~mL}$ of distilled water was sprinkled on the stomach wall before the initiation of ESD. Then, $20 \mathrm{~mL}$ of gastric juice was collected with a sterilized tube and submitted to a culture laboratory. Using an endoscope equipped with a water jet (GIF Q260J; Olympus, Tokyo, Japan), the stomach was irrigated with $2 \mathrm{~L}$ of saline throughout. After ESD was performed in the usual manner and resected tumors were collected, $20 \mathrm{~mL}$ of distilled water was sprinkled on the stomach wall. Then, $20 \mathrm{~mL}$ of gastric juice was collected with a sterilized tube and submitted to a culture laboratory. The opening of the forceps was sterilized with isodine, and the sterilized tube was inserted into the forceps before the tube was passed through the opening of the forceps. Fig. 1 shows the results of the effect on reducing the tota bacterial count. We compared changes in the quantity of cultured bacteria for gastric juice in a logarithmic representation. In the regular group, the bacterial count of gastric juice before ESD had a median of 6.45 (95\% confidence interval [CI], 4.93 to 7.32), and the bacterial count of gastric juice after ESD had a median of 5.62 (95\% CI, 3.86 to 6.64); there was no significant difference before and after ESD. In the clean group, the bacterial count of gastric juice before gastric lavage had a median of 6.50 (95\% CI, 3.88 to 8.11), and the bacterial count of gastric juice after ESD following gastric lavage had a median of 1.69 (95\% CI, 0.84 to 3.68); the quantity of cultured bacteria for gastric 
A

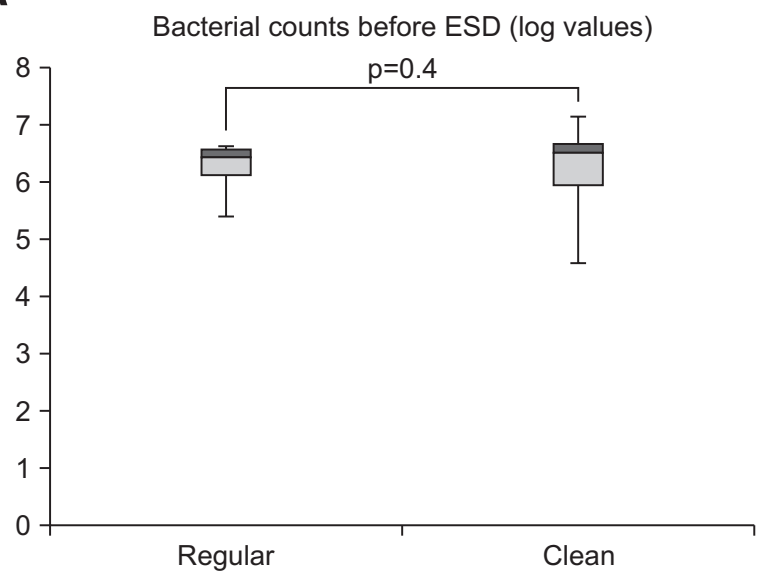

B

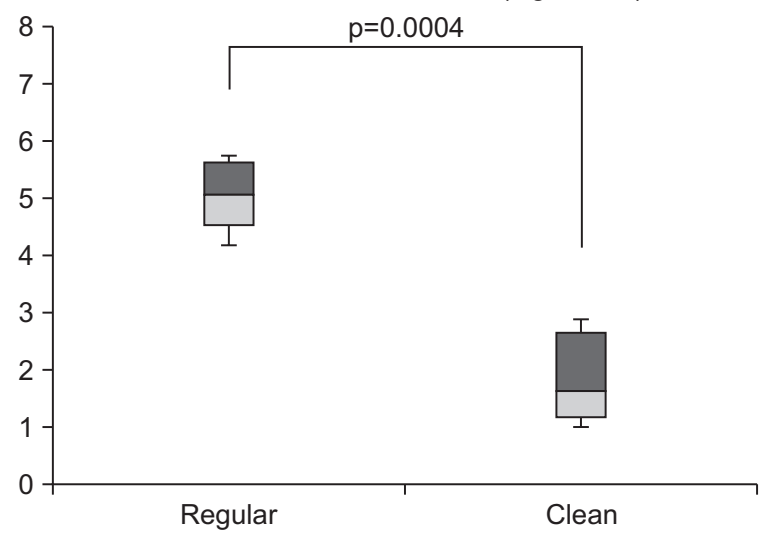

Fig. 1. (A, B) Comparison of logarithmic bacterial counts with and without saline irrigation before and after endoscopic submucosal dissection (ESD). The bacterial counts did not significantly differ between the regular group and the clean group before ESD ( $\mathrm{p}=0.4$ ). However, the difference in bacterial counts after ESD was significant between the regular group (without systemic irrigation) and the clean group (with systemic irrigation) $(\mathrm{p}=0.0004)$.

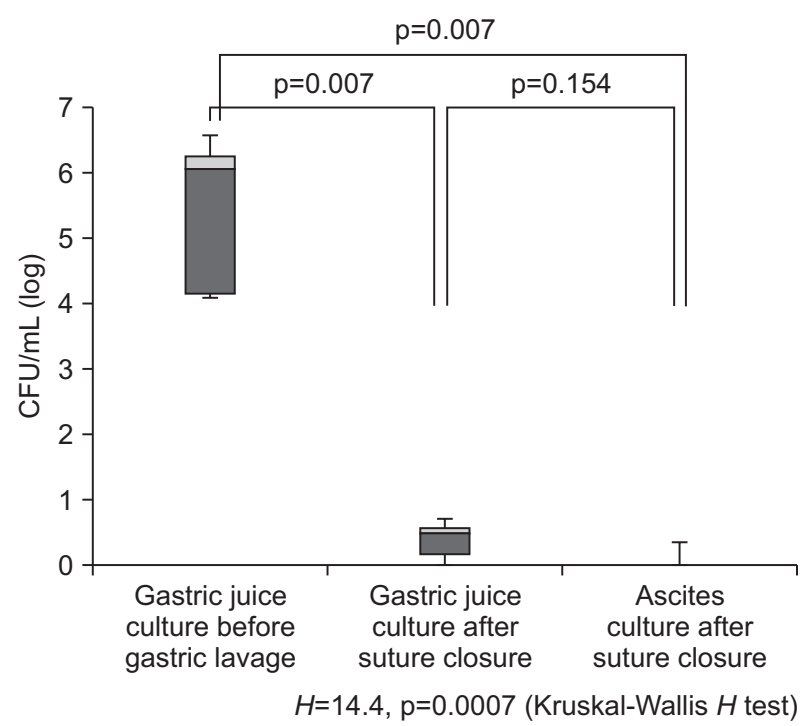

Fig. 2. Logarithmic bacterial counts before and after systematic lavage of the stomach in laparoscopy and endoscopy cooperation surgery. Although the bacterial counts in the gastric juice culture before gastric lavage and in the ascites culture after suture closure were significantly reduced ( $\mathrm{p}=0.007)$, no significant difference was observed between the bacterial counts in the gastric juice culture and the ascites culture after suture closure $(\mathrm{p}=0.154)$.

juice was significantly reduced by gastric lavage with 2,000 mL of saline ( $p=0.0004) .^{33}$ Based on these results, we examined laparoscopy and endoscopy cooperation surgery (LECS) in which the endoscope was exposed to the abdominal cavity. After the opening caused by full-thickness resection was sutured by hand, $20 \mathrm{~mL}$ of ascitic fluid was collected from the laparoscopic port and submitted to a culture laboratory. In addition, $20 \mathrm{~mL}$ of distilled water was sprinkled on the stomach wall. Subsequently, $20 \mathrm{~mL}$ of gastric juice was collected with a sterilized tube and submitted to a culture laboratory. The results demonstrate the effect on decreasing the total bacterial count for gastric juice after typical gastric lavage at the same level as ascitic fluid. For EFTR in the stomach, where gastric acid is present, it is thought that irrigation with saline may be more effective than disinfection with isodine, which may cause gastric mucosa injury. In 20 patients who underwent LECS, no postoperative infection was found after intravenous administration of antibiotics and irrigation of the upper gastrointestinal tract with $2 \mathrm{~L}$ of saline (Fig. 2). ${ }^{34}$ In conclusion, thorough intragastric irrigation with $2 \mathrm{~L}$ of saline or irrigation with saline combined with antibiotics provides a practical infection-control method by routing through the stomach, and this method can be introduced in clinical settings.

\section{3) Suturing of full-thickness wound after full-thickness excision (development of devices for flexible endoscopy and of full-thickness suturing device)}

For NOTES and EFTR, it was necessary to develop a reliable, multipurpose suturing device with suturing performance equivalent to hand-suturing surgical techniques.

Various full-thickness suturing devices have been developed and reported. Shortly after NOTES was first developed, a T-bar anchoring system was used for various experiments concerning full-thickness suturing. Seaman et al. ${ }^{35}$ verified various anchors using the following items: four tissue-anchor designs (T-bar, Tbar with mesh bolster, star, basket "T" bar [titanium, $1 \times 10 \mathrm{~mm}$ ]); a mesh bolster (polypropylene, $15 \times 50 \mathrm{~mm}$ ), held by a T-bar on the outside of the mesh; a "star" button (polyetheretherketon, 10-mm diameter); and a self-expanding hourglass-shaped double basket (nitinol, 10-mm diameter footprint). At the time of euthanasia, at 4 or 9 weeks, the numbers of retained anchors associated with intact plications for each group were as follows: T-bar, 6 of 9 pairs (67\%); mesh, 12 of 18 pairs (67\%); basket, 14 
of 18 pairs (78\%); and star, 2 of 9 pairs (22\%). Thus, the basket tissue anchors appear most promising. Although the T-bar system was simple, easy, and economical, it has the risk of injuring adjacent organs because of the use of a puncture needle; as a result, this design did not become popular.

For full-thickness suturing devices, the leak test has been used to evaluate sutures. Liu et al. ${ }^{36}$ prepared a 2-cm linear incision with 51 ex vivo porcine stomach models and evaluated technical difficulties of closure using Eagle Claw VIII and endoclips, as follows: bursting pressures were $56 \mathrm{~mm} \mathrm{Hg}$ (range, 35 to 110 $\mathrm{mm} \mathrm{Hg}$ ) for Eagle Claw VIII, $19 \mathrm{~mm} \mathrm{Hg}$ (range, 9 to $65 \mathrm{~mm}$ $\mathrm{Hg}$ ) for endoclips, and $78 \mathrm{~mm} \mathrm{Hg}$ (range, 63 to $110 \mathrm{~mm} \mathrm{Hg}$ ) for surgical suturing; eagle Claw VIII could withstand a higher endoluminal pneumatic bursting pressure than endoclips. Sun et al. ${ }^{37}$ measured the leakage pressures of endoclip, omentoplasty, over-the-scope-clip (OTSC), and hand-suturing and reported that suturing with OTSC and hand-suturing showed significantly higher suturing performance, as follows: the endoclip and omentoplasty groups generated similar leakage pressures $(34.5 \pm 2.6 \mathrm{~mm} \mathrm{Hg}$ vs $42.2 \pm 4.1 \mathrm{~mm} \mathrm{Hg}, \mathrm{p}>0.05)$, both lower than OTSC and hand-suturing groups $(81.5 \pm 2.1 \mathrm{~mm} \mathrm{Hg}$ and $87.0 \pm 3.0$ $\mathrm{mm} \mathrm{Hg}$, respectively, $\mathrm{p}<0.001)$. The only flexible endoscopic full-thickness suturing device with suturing threads that is currently available commercially (in the world) is the Overstitch System (Apollo Endosurgery, Austin, TX, USA) (Fig. 3A). ${ }^{38}$ A clip-type full-thickness suturing device, the OTSC (Ovesco Endoscopy GmbH, Tüebingen, Germany), is on the market (Fig. 3B). ${ }^{39}$ However, no full-thickness suturing device has been developed that can be easily attached to a common multipurpose flexible endoscope with one channel, and the Overstitch System
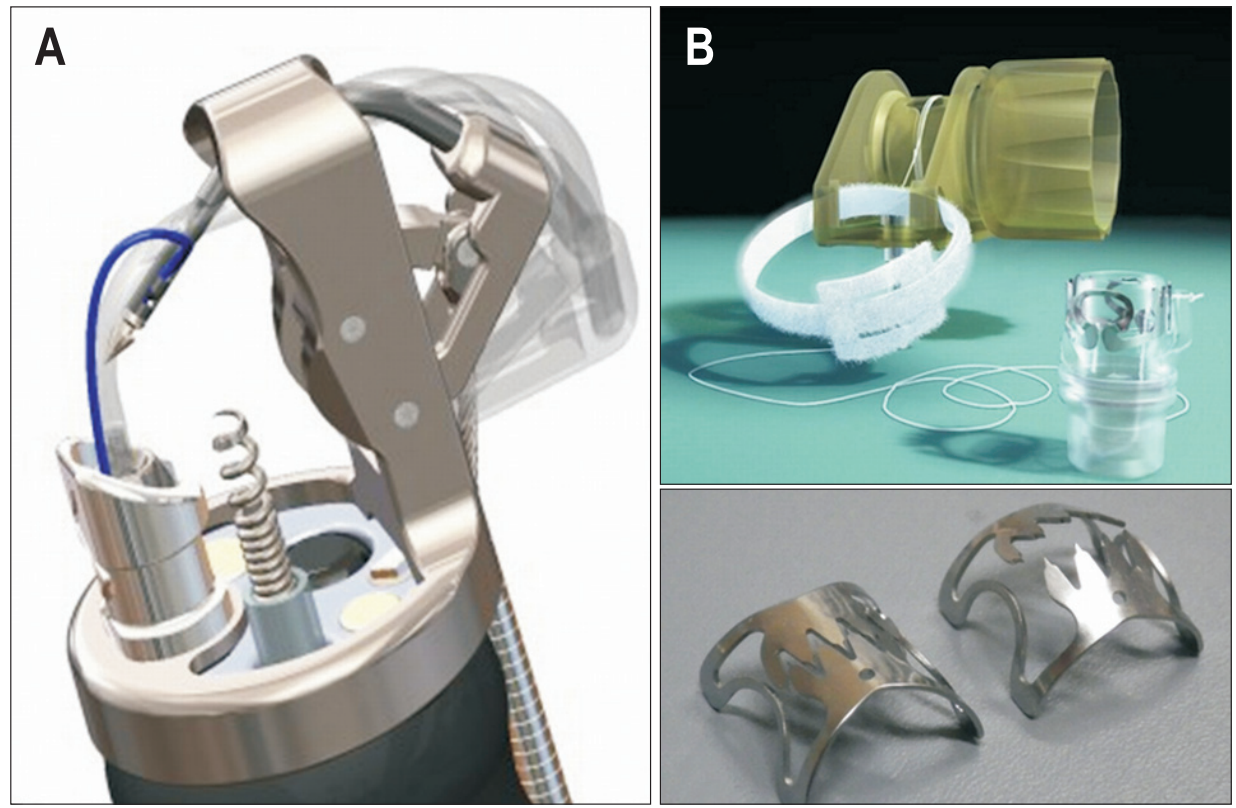

Fig. 3. Overstitch System and OverThe-Scope-Clip (OTSC). (A) Although the Overstitch System is an innovative flexible endoscopic full-thickness suturing device with suturing threads, because of its anteriorly directed suture needle, caution should be exercised so as not to cause injury to adjacent organs. Additionally, the OTSC requires two-channel scopes. (B) The OTSC can be attached to a normal multipurpose endoscope and can be used relatively easily. Because it is a clip-type suturing device, which means that it cannot turn back once it is released, caution should be exercised to avoid suture misfire.
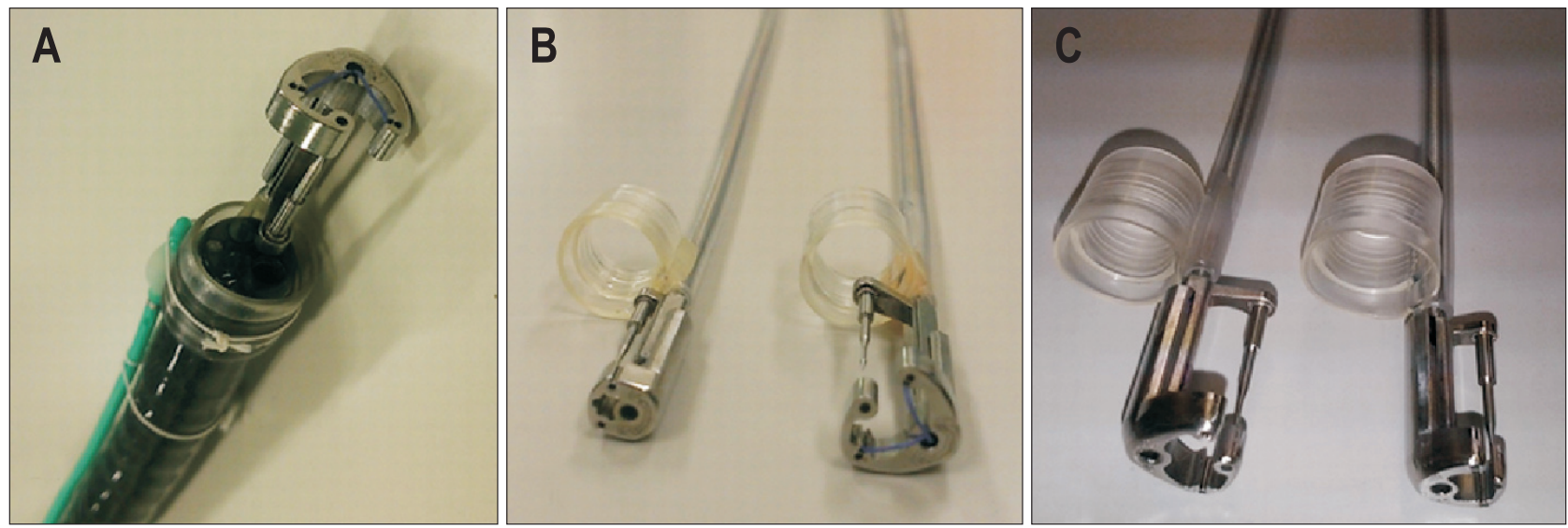

Fig. 4. Double-armed Bar Suturing System (DBSS). (A) The DBSS can be attached in a single operation by attaching the hood part to the tip of a multipurpose endoscope with one channel. (B, C) Various types of the DBSS, such as the Mini-DBSS, the Middle-DBSS, and the Normal-DBSS, can be selected according to the size of the perforation opening. 
requires two channel scopes.

Although the Overstitch System is an innovative flexible endoscopic full-thickness suturing device with suturing threads, its anteriorly-directed suture needle may cause injury and suturing of adjacent organs when the full-thickness stomach wall is sutured. The OTSC can be attached to a normal multipurpose endoscope and be used with relative ease. However, it has a problem with suture misfire because it is a clip-type suturing device, meaning it cannot be turned back once it is released. Furthermore, it is mechanical and does not use any suturing thread, which causes gaps in the OTSC intervals, making it difficult to complete full-thickness suturing. Thus, there remains no flexible endoscopic full-thickness suturing device that is economical, simple, easily operated, and can be attached to multipurpose endoscopes. Mori et al. ${ }^{40}$ developed a flexible endoscopic full-thickness suturing device which provides suturing performance comparable to surgical hand-suturing techniques. For the Double-armed Bar Suturing System (DBSS), as shown in Fig. 4A and B, only a hood is attached to the tip of the multipurpose, one-channel endoscope, and the opening of the forceps is not used. Thus, opening of the forceps may yet have various uses for the DBSS (Fig. 4A). Based on the size of the perforation opening, various prototypes, such as Mini-DBSS, Middle-DBSS, and Normal-DBSS, can be used (Fig. 4B and C).

Mori et $a l^{40}$ used DBSS prototypes to conduct many studies on full-thickness suturing of resected stomachs in swine. In one of their fundamental experiments, they used an air-leak test to examine whether the prototypes showed suturing performances equivalent to surgical hand-suturing techniques. A leak test of the wound caused by full-thickness excision after EFTR (in swine resected stomach) was performed every 10 sections, and there was no significant difference between surgical handsuturing and DBSS, as follows ( $\mathrm{p}=0.542)$ : for surgical hand-suturing, the median was 3,350 Pa (G), and 95\% CI, 2,470 to 4,250 $\mathrm{Pa}(\mathrm{G})$; for DBSS, the median was 3,665 Pa (G), and 95\% CI, 1,600 to $4,400 \mathrm{~Pa}(\mathrm{G})$. Using this full-thickness suturing device, for which suturing performance can be appropriately adjusted and changed, full-thickness perforation can be sutured with absorbable thread. In an experimental animal (dog), after resection of the full-thickness virtual stomach lesion (40 mm) (Fig. 5A and B), a 4-mm bite and 4-mm pitch were sutured (Fig. 5C), after which the wound was successfully closed with 10 stitches, as shown in Fig. 5D. In such in vivo animal experiments with dogs, survival of 1 year was achieved, and safety was confirmed. ${ }^{41}$ Development of a full-thickness suturing device that can be used for multipurpose flexible endoscopy may yet be advanced further.

\section{4) Expansion of a surgical field for gastrointestinal-tract collapse caused by perforation (development of counter traction device)}

Regarding NOTES-related procedures, one reported study explores a method of securing a surgical field for gastrointestinaltract collapse caused by perforation during EFTR. In this report, a stomach lifting method was applied via stomach-wall fixation,
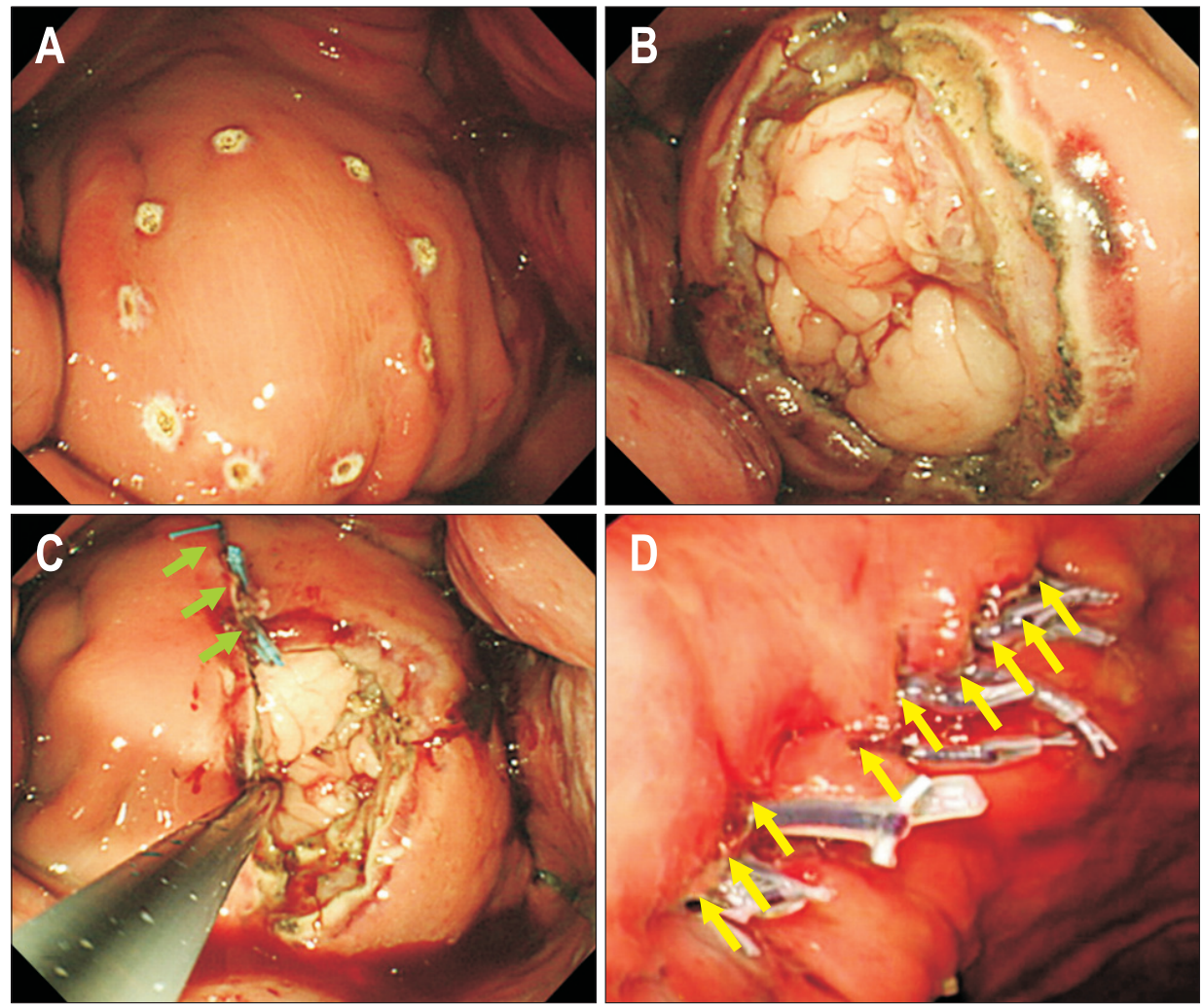

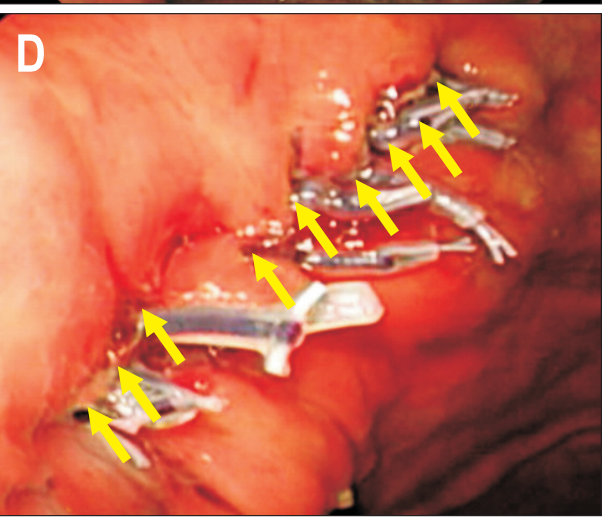

Fig. 5. In vivo animal experiments using pure endoscopic full-thickness resection and full-thickness suturing. (A) An area $40 \mathrm{~mm}$ in diameter was marked as a simulated tumor. (B) Using the Mechanical Counter-Traction System, which is housed inside an overtube, the collapsing stomach wall was extended with eight expansion arms, and a 40-mm-diameter virtual tumor was fully resected. (C) After full-thickness resection, singlenodule full-thickness suturing of the 4-mm bite and 4-mm pitch was performed. Three stitches had been done (green arrows). (D) Ten stitches were put in the wound (yellow arrows), and the experimental dog survived without complications for 1 year after surgery. 
which is used to create additional gastric fistula. ${ }^{42}$ However, there have been no reports regarding attaching a counter traction device to a flexible endoscope.

Although Cai et al. ${ }^{43}$ reported EFTR use for a benign submucosal tumor of the colon, colon collapse during pretreatment and during resection of the full-thickness tumor was not discussed in detail. In addition, two of the four patients experienced stomachache, fever, and peritonitis after surgery. In comparison with the upper gastrointestinal tract, colon EFTR necessitates a systematic irrigation method, full-thickness suturing device, and counter traction device. Elmunzer et al. ${ }^{44}$ reported that EFTR was performed by pulling the gastric mucosa using an anchor and conducting a full-thickness resection with a snare. However, expansion of a surgical field during suturing of the full-thickness wound was not mentioned. Zhou et al. ${ }^{45}$ reported the successful use of EFTR without laparoscopic assistance to resect 26 gastric submucosal tumor cases originating from the muscularis propria layer. Their EFTR technique is derived from the standard ESD and closure of the gastric wall defect with several metallic clips. Although the mean tumor diameter was $28 \mathrm{~mm}$ and the opening after resection seemed to exceed $30 \mathrm{~mm}$, there was no clear explanation regarding expansion of the surgical field for gastrointestinal-tract collapse when a clip was closed after tumor resection. In our verification of the animal experiment, even OTSC showed lower suturing strength than full-thickness suturing with suturing thread. It seems that from the viewpoints of safety and reliability, the opening after resection cannot be closed using normal clips. In EFTR without the support of a laparoscope, the full-thickness suturing device and counter traction device are thought to be an important pair.

We developed and reported various prototypes of counter traction devices. ${ }^{46,47}$ Fig. $6 \mathrm{~A}$ and B show the Mechanical Counter Traction System (MCTS) that is housed inside an overtube. Fig. 6C shows EFTR of a 40-mm-diameter virtual lesion in resected swine stomach using a prototype of MCTS. Stopping insufflation of the endoscope, the control part at hand was pushed out, the collapsing stomach wall was extended with eight expansion arms, and the full-thickness stomach wall of the site (40-mmdiameter virtual tumor) was resected. However, there were limitations in this overtube-type MCTS regarding compliance with the complicated endoscopic operation, such as the reversing operation. Thus, we intentionally placed balloons, which ex-
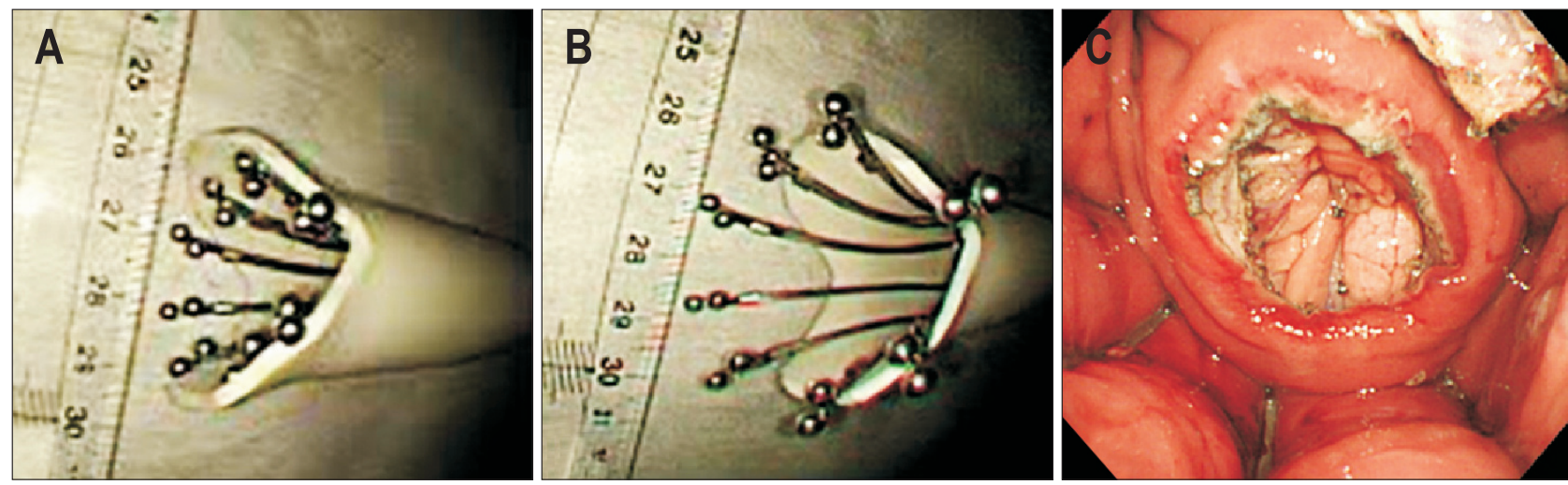

Fig. 6. Mechanical Counter-Traction System (MCTS). (A) The MCTS is housed inside an overtube with a 20-mm diameter. (B) When the overtube is pulled, eight expansion arms widen the collapsing stomach wall. (C) In an in vivo experiment in a dog, sufficient expansion of the surgical field allowed endoscopic full-thickness resection to be accomplished without insufflation.
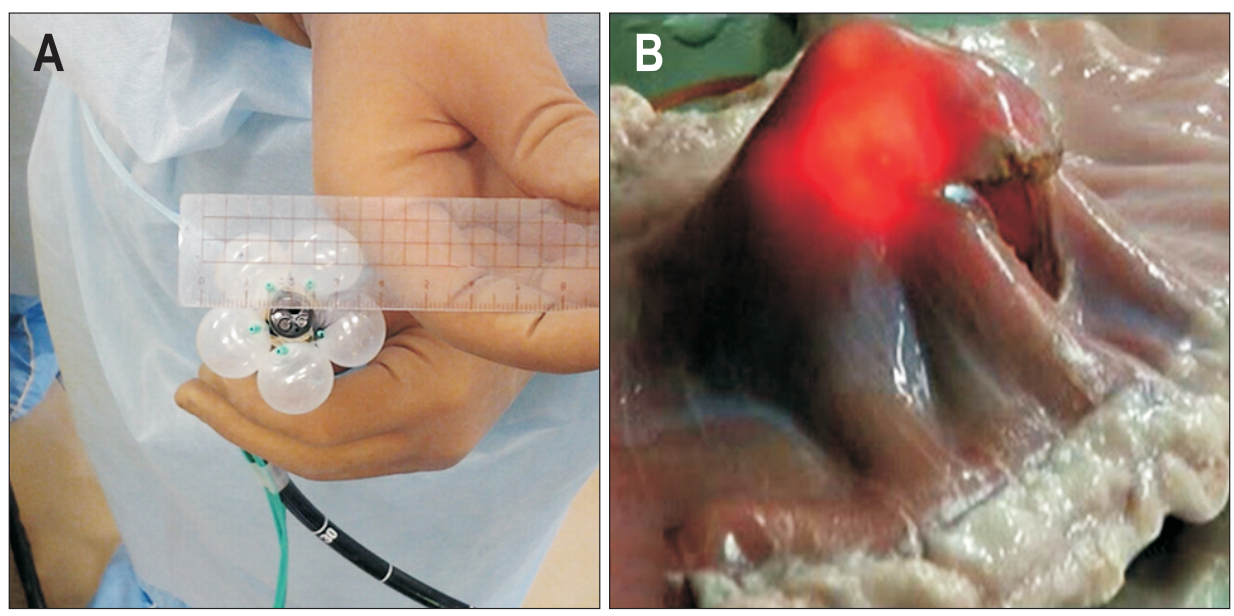

Fig. 7. Balloon Arm-Mechanica Counter-Traction System (BA-MCTS). (A) This is a balloon-type countertraction device that uses a large number of single-side expanded balloons. The expansion ability for a collapsing stomach wall is slightly inferior to that of MCTS, but its operability is acceptable. (B) Expansion of a surgical field is enabled in any part of the excised porcine stomach without insufflation, even in the reversed position. 
pand on one side on the tip of the endoscope body, so that they would interfere with each other, to avoid blocking the visual endoscopic field. Fig. 7 is a balloon-type counter traction device that uses a large number of one-side expanded balloons. This Balloon Arm-Mechanical Counter Traction System (BA-MCTS) is attached to the tip of the endoscope (Fig. 7A) and enables expansion of the surgical field, such as the reversing operation of the entire stomach without insufflation. Fig. 7B shows pure EFTR in an excised porcine stomach.

\section{5) Monitoring of abdominal air pressure by insufflation (de-} velopment of pressure monitoring system)

In intestinal endoscopy, endoscopists perform aspiration and insufflation manually. Although pressure in the gastrointestinal tract is estimated based on the operator's subjective view, objective data pertaining to abdominal air pressure is necessary to ensure safe EFTR. Nakajima et $a l^{48}$ and Kato et al. ${ }^{49}$ were the first to report the use of a constant pressure device for

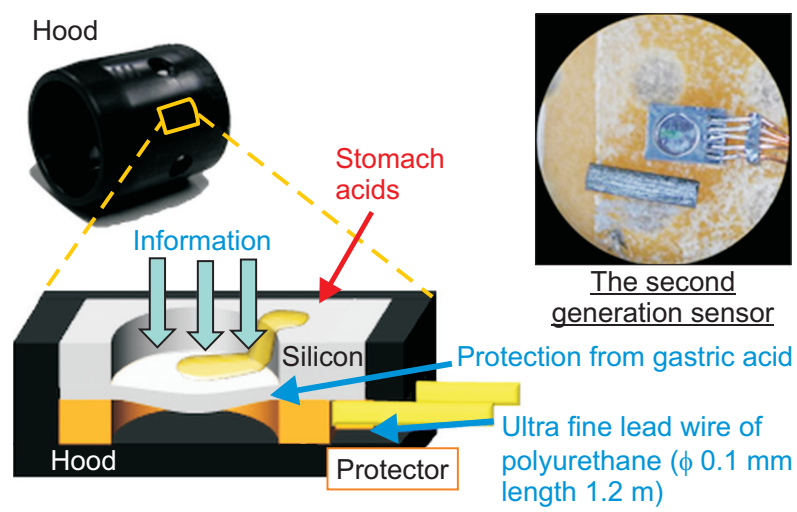

Fig. 8. Pressure signal-capturing structure of the Micro Electro Mechanical System pressure sensor hood. Even under strongly acidic conditions, such as in gastric juice, the pressure of the tip of the endoscope can be measured in real time. flexible endoscopy. An overtube inserted into the esophagus goes through the Leak Cutter ${ }^{\circledR}$ (2F09A; TOP Co., Tokyo, Japan), which is a connector with a pneumoperitoneum apparatus and is connected to the extracorporeal pneumoperitoneum apparatus to maintain a constant specific gastrointestinal pressure. This is an innovative device that enables treatment under a specific pressure even if insufflation or aspiration is not performed by an operator; as such, it is a concept similar to the pneumoperitoneum trocker of a laparoscope. ${ }^{48,49}$ The Phase I study using this device has been conducted, and favorable results have been reported.

We developed a hood equipped with a semiauto constant pressure device that is economical and has a simple structure. The hood equipped with the Micro Electro Mechanical System (MEMS) pressure sensor can measure the pressure of the tip of the endoscope in real time (Fig. 8). Based on the animal experiment, the MEMS sensor was downsized to $2 \mathrm{~mm}$ in width and $0.8 \mathrm{~mm}$ in thickness and implemented in the hood (Fig. 9A). The MEMS pressure sensor does not block the visual field of the endoscope at all (Fig. 9B), and the extrafine, 0.1-mm-diameter polyurethane conducting did not affect operation of the endoscope (Fig. 9C). In the in vivo dog experiment, there was a good correlation between the direct pressure from the percutaneous endoscopic gastrostomy and the electric signal from the MEMS hood. The compression and depression test (aspiration and insufflation test) was performed consecutively five times and showed an increase and decrease in internal pressure over time. Of five tests, we analyzed the results obtained from the four tests that resulted in accurate measurable data. There was a good correlation between the pressure-monitoring sensor ( $\mathrm{mm}$ $\mathrm{Hg}$ ) and the signal strength (V) of the MEMS hood. The signal strengths (in these four tests) ranged from -0.01 to $0.138 \mathrm{~V}$ and were, thus, well correlated with the actual pressure, which measured from 0 to $22 \mathrm{~mm} \mathrm{Hg}$ via the pressure monitoring sensor
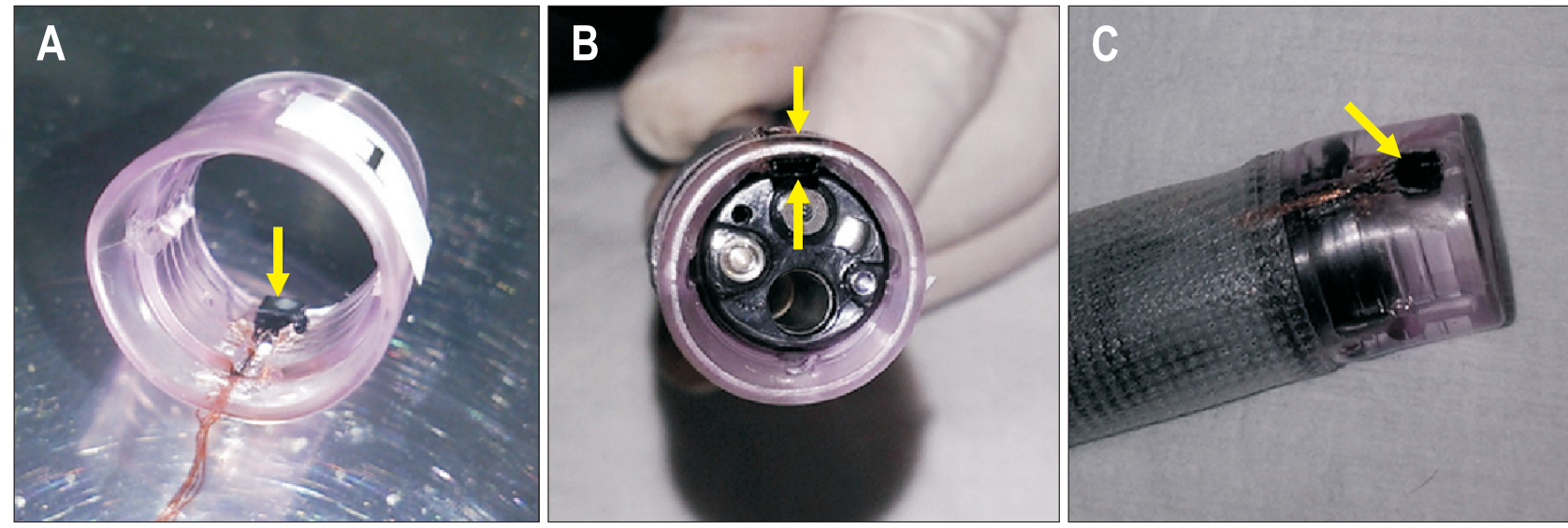

Fig. 9. Hood equipped with Micro Electro Mechanical System (MEMS) pressure sensor. (A) The MEMS sensor was downsized to 2 mm in width and $0.8 \mathrm{~mm}$ in thickness and was implemented in the hood. (B) The MEMS pressure sensor does not block any of the visual field of the endoscope. (C) The extrafine, 0.1-mm-diameter polyurethane conducting wire did not affect operation of the endoscope. All yellow arrows show a tiny MEMS pressure sensor attached to the hood. 


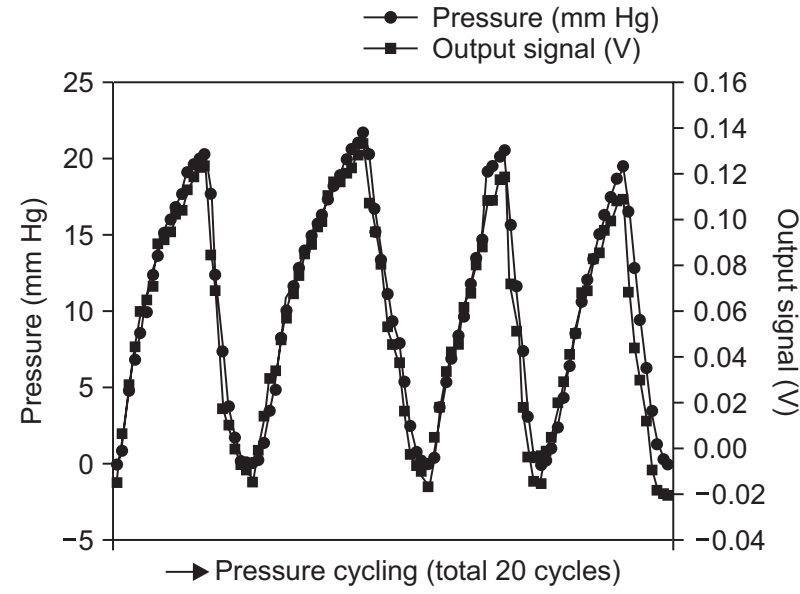

Fig. 10. Relationship between output signals and directly measured pressures. In an in vivo experiment performed in dogs, there was a good correlation between the direct pressure from percutaneous endoscopic gastrostomy and the electrical signal from the Micro Electro Mechanical System hood.

(Fig. 10). Only by attaching the MEMS hood to the endoscope is it possible to measure intragastric pressure in real time. ${ }^{50}$

\section{6) Training and future perspective of EFTR}

The development of NOTES and EFTR includes not only the medical aspects but also the legal and technical aspects pertaining to the development of a new device; thus, development solely by medical practitioners has its limits. Regarding medical aspects, the ESD skill level of Japanese endoscopists, which may vary depending on institutions, is very high. However, the following problems are inevitable:

(1) As basic techniques of EFTR, expertise in incision, abrasion, and hemorrhage control using a flexible endoscope is essential. It is necessary for gastroenterological endoscopists to recognize the particularities of this procedure, which is not included in previous intracavitary therapies (treatment and surgery are performed in the abdominal cavity from a semi-closed system and the lumen), and to establish operative procedures to ensure that the procedure can be performed effectively and safely. The current knowledge of gastroenterological endoscopists is insufficient to perform EFTR in clinical settings. It is essential that such endoscopists understand the surgical anatomy of the organs located on the other side of the stomach wall that is being resected and to acquire basic surgical techniques for device development. This is similar to the requirement that endoscopists who perform ESD become familiar with the anatomy of the interior of the stomach wall.

(2) On the other hand, rigid endoscopy still plays a role with surgeons. Surgeons are well trained in telescopic techniques in which torque is directly transmitted. This field has been advanced in laparoscopic surgery. On the other hand, there are particularities in NOTES-related procedures, such as EFTR. These procedures mainly use a flexible endoscope, which moves in a very particular, paradoxical way. Basically, the surgery must be conducted using a single endoscope; thus, surgeons have to perform complicated incision, abrasion, hemorrhage control, and ligation using an unfamiliar flexible endoscope. Unless both endoscopists and surgeons are willing to learn their respective lacking expertise, it is impossible to establish safe EFTR.

(3) With regard to legal concerns, as a matter of course, preparations of ethical aspects are required for the development of devices. It goes without saying that approval of in-hospital ethical committee is required. In addition, various engineering knowledge and legal preparations, including cooperation with various companies, are necessary for the development of new devices. However, physicians typically know little about these fields and, therefore, do not possess the capacity to learn and become familiar with these fields. Thus, it is necessary to establish a system of comprehensive EFTR development by which physicians closely cooperate with experts of various domains in intellectual property and/or patent law.

\section{CONCLUSIONS}

Laparoscopy and flexible endoscopy are very similar endoscopic operations once the surgical anatomy has been learned. Nonetheless, it is necessary to establish an endoscopic treatment and surgery department that specializes in endoscopic surgery. It is true that there are many more discoveries that can be applied to EFTR and NOTES as well as intraluminal treatment. In particular, gastrointestinal tumors can be approached by the shortest distance from the mouth using a flexible endoscope, making EFTR the surgical procedure with the lowest degree of invasion. There are certainly difficult sites for EFTR, but those sites can be covered by laparoscopic procedures that are virtually identical to endoscopy. The established advantages of laparoscopic surgery will be inherited by this endoscopic technique, and the procedural method can be altered if situations more suitable to EFTR and NOTES are found at the research and development stage and if theoretical, technical, and ethical problems are resolved. There are many merits in extremely minimally invasive surgery, such as reducing the burden (financial, physical, and mental) on patients well as medical costs. Even with the realization and increased use of surgery that requires capsule endoscopy, perfecting a robot or the required expensive machine parts will still take time. Therefore, developing a new surgical method that uses existing multipurpose flexible endoscopic methods, such as EFTR and NOTES, constitutes a positive contribution to society and should be urgently encouraged for the time being.

\section{CONFLICTS OF INTEREST}

Drs Hirohito Mori, Hideki Kobara, Noriko Nishiyama, Shintaro Fujihara, and Tsutomu Masaki have no conflicts of interest 
and no financial arrangement with any company, institution, or third party.

\section{ACKNOWLEDGEMENTS}

The authors acknowledge the Kagawa NOTES Project for supporting this study.

\section{REFERENCES}

1. Ono H, Kondo H, Gotoda T, et al. Endoscopic mucosal resection for treatment of early gastric cancer. Gut 2001;48:225-229.

2. Fujishiro M, Yahagi N, Kakushima N, et al. Endoscopic submucosal dissection of esophageal squamous cell neoplasms. Clin Gastroenterol Hepatol 2006;4:688-694.

3. Yamamoto H, Kawata H, Sunada K, et al. Successful en-bloc resection of large superficial tumors in the stomach and colon using sodium hyaluronate and small-caliber-tip transparent hood. Endoscopy 2003;35:690-694.

4. Kalloo AN, Singh VK, Jagannath SB, et al. Flexible transgastric peritoneoscopy: a novel approach to diagnostic and therapeutic interventions in the peritoneal cavity. Gastrointest Endosc 2004; 60:114-117.

5. Yamamoto M, Zaima M, Yamamoto H, Harada H, Kawamura J, Yamaguchi T. A modified overlap method using a linear stapler for intracorporeal esophagojejunostomy after laparoscopic total gastrectomy. Hepatogastroenterology 2014;61:543-548.

6. Lacerda CF, Bertulucci PA, Oliveira AT. Step-by-step esophagojejunal anastomosis after intra-corporeal total gastrectomy for laparoscopic gastric cancer treatment: technique of "reverse anvil". Arq Bras Cir Dig 2014;27:71-76.

7. Brigic A, Southgate A, Sibbons P, Clark SK, Fraser C, Kennedy RH. Full-thickness laparoendoscopic colonic excision in an experimental model. Br J Surg 2013;100:1649-1654.

8. Aggarwal S, Sharma AP, Ramaswamy N. Outcome of laparoscopic sleeve gastrectomy with and without staple line oversewing in morbidly obese patients: a randomized study. J Laparoendosc Adv Surg Tech A 2013;23:895-899.

9. Machado MA, Surjan RC, Makdissi FF. First single-port laparoscopic liver resection in Brazil. Arq Bras Cir Dig 2013;26:144-146.

10. Petersen TI, Pahle E, Sommer T, Zilling T. Laparoscopic minimally invasive total gastrectomy with linear stapled oesophagojejunostomy: experience from the first thirty procedures. Anticancer Res 2013;33:3269-3273.

11. Chong-Wei K, Dan-Lei C, Dan D. A modified technique for esophagojejunostomy or esophagogastrostomy after laparoscopic gastrectomy. Surg Laparosc Endosc Percutan Tech 2013;23:e109e115.

12. Yasuda K, Kitano S, Ikeda K, Sumiyama K, Tajiri H. Assessment of a manipulator device for NOTES with basic surgical skill tests: a bench study. Surg Laparosc Endosc Percutan Tech 2014;24:e191e195.
13. Liu L, Chiu PW, Teoh AY, Lam CC, Ng EK, Lau JY. Endoscopic suturing is superior to endoclips for closure of gastrotomy after natural orifices translumenal endoscopic surgery (NOTES): an ex vivo study. Surg Endosc 2014;28:1342-1347.

14. Grund KE, Lehmann TG. Transesophageal NOTES: a critical analysis of relevant problems. Minim Invasive Ther Allied Technol 2010;19:252-256.

15. Ye LP, Yu Z, Mao XL, Zhu LH, Zhou XB. Endoscopic full-thickness resection with defect closure using clips and an endoloop for gastric subepithelial tumors arising from the muscularis propria. Surg Endosc 2014;28:1978-1983.

16. Abe N, Takeuchi H, Ooki A, et al. Recent developments in gastric endoscopic submucosal dissection: towards the era of endoscopic resection of layers deeper than the submucosa. Dig Endosc 2013;25 Suppl 1:64-70

17. von Renteln D, Rösch T, Kratt T, Denzer UW, El-Masry M, Schachschal G. Endoscopic full-thickness resection of submucosal gastric tumors. Dig Dis Sci 2012;57:1298-1303.

18. Elmunzer BJ, Trunzo JA, Marks JM, et al. Endoscopic fullthickness resection of gastric tumors using a novel grasp-andsnare technique: feasibility in ex vivo and in vivo porcine models. Endoscopy 2008;40:931-935.

19. von Renteln D, Riecken B, Walz B, Muehleisen H, Caca K. Endoscopic GIST resection using FlushKnife ESD and subsequent perforation closure by means of endoscopic full-thickness suturing. Endoscopy 2008;40 Suppl 2:E224-E225.

20. Nakajima K, Nishida T, Takahashi T, et al. Partial gastrectomy using natural orifice translumenal endoscopic surgery (NOTES) for gastric submucosal tumors: early experience in humans. Surg Endosc 2009;23:2650-2655.

21. Suzuki H, Ikeda K. Endoscopic mucosal resection and full thickness resection with complete defect closure for early gastrointestinal malignancies. Endoscopy 2001;33:437-439.

22. Ryou M, Hazan R, Rahme L, Thompson CC. An ex vivo bacteriologic study comparing antiseptic techniques for natural orifice translumenal endoscopic surgery (NOTES) via the gastrointestinal tract. Dig Dis Sci 2012;57:2130-2136.

23. Wittmann DH, Condon RE. Prophylaxis of postoperative infections. Infection 1991;19 Suppl 6:S337-S344.

24. Moine P, Fish DN. Pharmacodynamic modelling of intravenous antibiotic prophylaxis in elective colorectal surgery. Int J Antimicrob Agents 2013;41:167-173.

25. Nahlieli O, Baruchin AM, Levi D, Shapira Y, Yoffe B. Povidoneiodine related burns. Burns 2001;27:185-188.

26. Liu FC, Liou JT, Hui YL, et al. Chemical burn caused by povidoneiodine alcohol solution: a case report. Acta Anaesthesiol Sin 2003; 41:93-96.

27. Demir E, O’Dey DM, Pallua N. Accidental burns during surgery. J Burn Care Res 2006;27:895-900.

28. Hesami MA, Alipour H, Nikoupour Daylami H, Alipour B, Bazargan-Hejazi S, Ahmadi A. Irrigation of abdomen with imipenem solution decreases surgical site infections in patients with perfo- 
rated appendicitis: a randomized clinical trial. Iran Red Crescent Med J 2014;16:e12732.

29. Nikfarjam M, Weinberg L, Fink MA, et al. Pressurized pulse irrigation with saline reduces surgical-site infections following major hepatobiliary and pancreatic surgery: randomized controlled trial. World J Surg 2014;38:447-455.

30. Sodergren MH, Pucher P, Clark J, et al. Disinfection of the access orifice in NOTES: evaluation of the evidence base. Diagn Ther Endosc 2011;2011:245175.

31. Memark VC, Anderson JB, Nau PN, et al. Transgastric endoscopic peritoneoscopy does not lead to increased risk of infectious complications. Surg Endosc 2011;25:2186-2191.

32. Narula VK, Happel LC, Volt K, et al. Transgastric endoscopic peritoneoscopy does not require decontamination of the stomach in humans. Surg Endosc 2009;23:1331-1336.

33. Mori H, Kobara H, Rafiq K, et al. Effects of gastric irrigation on bacterial counts before endoscopic submucosal dissection: a randomized case control prospective study. PLoS One 2013;8:e65377.

34. Mori H, Kobara H, Tsushimi T, et al. Reduction effect of bacterial counts by preoperative saline lavage of the stomach in performing laparoscopic and endoscopic cooperative surgery. World J Gastroenterol 2014;20:15763-15770.

35. Seaman DL, Gostout CJ, de la Mora Levy JG, Knipschield MA. Tissue anchors for transmural gut-wall apposition. Gastrointest Endosc 2006;64:577-581.

36. Liu L, Chiu PW, Teoh AY, Lam CC, Ng EK, Lau JY. Endoscopic suturing is superior to endoclips for closure of gastrotomy after natural orifices translumenal endoscopic surgery (NOTES): an ex vivo study. Surg Endosc 2014;28:1342-1347.

37. Sun G, Yang Y, Zhang X, et al. Comparison of gastrotomy closure modalities for natural orifice transluminal surgery: a canine study. Gastrointest Endosc 2013;77:774-783.

38. Kantsevoy SV, Thuluvath PJ. Successful closure of a chronic refractory gastrocutaneous fistula with a new endoscopic suturing device (with video). Gastrointest Endosc 2012;75:688-690.

39. von Renteln D, Vassiliou MC, Rothstein RI. Randomized controlled trial comparing endoscopic clips and over-the-scope clips for closure of natural orifice transluminal endoscopic surgery gastrotomies. Endoscopy 2009;41:1056-1061.

40. Mori H, Kobara H, Fujihara S, et al. Feasibility of pure EFTR using an innovative new endoscopic suturing device: the Double-armbar Suturing System (with video). Surg Endosc 2014;28:683-690.

41. Mori H, Kobara H, Kazi R, Fujihara S, Nishiyama N, Masaki T. Balloon-armed mechanical counter traction and double-armed bar suturing systems for pure endoscopic full-thickness resection. Gastroenterology 2014;147:278-280.e1.

42. Ikeda K, Sumiyama K, Tajiri H, Yasuda K, Kitano S. Evaluation of a new multitasking platform for endoscopic full-thickness resection. Gastrointest Endosc 2011;73:117-122.

43. Cai MY, Zhong YS, Zhou PH, Xu MD, Yao LQ. Feasibility of endoscopic full-thickness resection in the treatment of colorectal submucosal tumors. Zhonghua Wei Chang Wai Ke Za Zhi 2012;15: 679-681.

44. Elmunzer BJ, Trunzo JA, Marks JM, et al. Endoscopic fullthickness resection of gastric tumors using a novel grasp-andsnare technique: feasibility in ex vivo and in vivo porcine models. Endoscopy 2008;40:931-935.

45. Zhou PH, Yao LQ, Qin XY, et al. Endoscopic full-thickness resection without laparoscopic assistance for gastric submucosal tumors originated from the muscularis propria. Surg Endosc 2011;25:2926-2931.

46. Mori H, Rafiq K, Kobara H, et al. Development of pure endoscopic full-thickness resection with mechanical countertraction and double-armed bar suturing systems. Gastrointest Endosc 2014;79:2425.

47. Mori H, Rafiq K, Kobara H, et al. Innovative noninsufflation EFTR: sufficient endoscopic operative field by mechanical counter traction device. Surg Endosc 2013;27:3028-3034.

48. Nakajima K, Moon JH, Tsutsui S, et al. Esophageal submucosal dissection under steady pressure automatically controlled endoscopy (SPACE): a randomized preclinical trial. Endoscopy 2012;44: 1139-1148.

49. Kato M, Nakajima K, Yamada T, et al. Esophageal submucosal dissection under steady pressure automatically controlled endoscopy (SPACE): a clinical feasibility study. Endoscopy 2014;46:680-684.

50. Mori H, Takao H, Kobara H, et al. Precise tumor size measurement under constant pressure by novel real-time micro-electromechanical-system hood for proper treatment (with videos). Surg Endosc 2015;29:212-219. 